\title{
Duality of Gauß-Manin systems associated to linear free divisors
}

\author{
Christian Sevenheck
}

June 4, 2018

\begin{abstract}
We investigate differential systems occurring in the study of particular non-isolated singularities, the so-called linear free divisors. We obtain a duality theorem for these $\mathcal{D}$-modules taking into account filtrations, and deduce degeneration properties of certain Frobenius manifolds associated to linear sections of the Milnor fibres of the divisor.
\end{abstract}

\section{Introduction}

The aim of the present note is to study the duality theory of some particular differential systems, which were introduced in [GMS09]. These are Gauß-Manin systems associated to hyperplane sections of Milnor fibres of special non-isolated singularities, called linear free divisors. These Gauß-Manin systems are used in loc.cit. for the construction of families of Frobenius manifolds (following the general framework from [DS03]), which generalizes the Frobenius structure defined Gy the quantum cohomology of the projective space. A natural question one may ask in this context is whether they also arise as the quantum cohomology of some variety (or orbifold). A particular and interesting class of examples rof linear free divisors are discriminants in representation spaces of a quiver $Q$ (see, e.g., [BM06] and [GMNS09]). In that case, one may also ask about the relationship between the Frobenius structures constructed in loc.cit. and The conjectured Frobenius manifold structure on the space of stability conditions in $D^{b}(\bmod k Q)$ (see ,e.g., [Bri09] or Tak05]). In any case, a precise study of the various properties of linear free divisors and of the Frobenius manifolds from [GMS09] is of interest in both singularity theory and representation theory. Particularly important is a detailed understanding of the degeneration behavior at the limit point of the parameter space (corresponding to the hyperplane Section of $D$ itself, and generalizing the large radius limit of the quantum cohomology of $\mathbb{P}^{n}$ ). Some questions on the limit behavior of the Frobenius manifolds constructed in [GMS09] remained open in that paper because of a lack of understanding of the duality theory of the Gauß-Manin systems associated to the above mentioned hyperplane jections. In this paper, we prove a conjecture from loc.cit. and give some consequences on this degeneration behavior. The basic tool for this proof is an explicit description of the Gauß-Manin system by differential operators, for which the duality statements needed can be calculated directly.

Zlet us notice that the mirror of the quantum cohomology of the projective spaces can also be generalized by the -mirror Landau-Ginzburg model of a weighted projective space, in that case, one can similarly study the associated filtered Gauß-Manin systems, and due to the more explicit control of the relevant cohomological invariants (the spectral numbers, see proposition 3 below), the corresponding statements have been shown in [DM09], following general results on a purely algebraic construction of Frobenius manifolds in [Dou09]. The main point in this note is to obtain these results for the Gauß-Manin systems of linear sections of Milnor fibres of linear free divisors, where due to the more complicated combinatorial structure of the input data (like quiver representations) the distribution of the spectral numbers is less easy to control.

Acknowledgements: I would like to thank Thomas Reichelt and John Alexander Cruz Morales for interesting discussions on topics related to this article.

\section{Linear free divisors, hyperplane sections and Gauß-Manin systems}

We start by introducing the main objects of interest of this paper. We also give a short but self-contained account of the results from [GMS09] needed here. More details can be found in loc.cit. and in [Sev11].

2000 Mathematics Subject Classification. 32S40, 34M35.

Keywords: Frobenius manifold, linear free divisors, spectral numbers, Brieskorn lattice, Birkhoff problem.

This research is supported by a DFG Heisenberg fellowship (Se 1114/2-1) and by ANR grant ANR-08-BLAN-0317-01 (SEDIGA). 
Definition-Lemma 1. Write $V$ for the affine space $\mathbb{C}^{n}$ with coordinates $x_{1}, \ldots, x_{n}$. Let $D \subset V$ be a reduced hypersurface, given by a polynomial equation $h \in \mathcal{O}_{V}$.

1. $D$ is called a linear free divisor iff there is a basis $\vartheta_{1}, \ldots, \vartheta_{n}$ of the $\mathcal{O}_{V}$-module $\Theta(-\log D):=\left\{\vartheta \in \Theta_{V} \mid \vartheta(h) \subset\right.$ $(h)\}$ (in particular, $\Theta(-\log D)$ must be $\mathcal{O}_{V}$-free) such that $\vartheta_{i}=\sum_{i=1}^{n} a_{i j} \partial_{x_{j}}$ where $a_{i j} \in \mathbb{C}[V]_{1}$ is a linear form. We will also consider the submodule $\Theta(-\log h)=\{\vartheta \in \Theta(-\log D) \mid \vartheta(h)=0\} . \Theta(-\log h)$ is $\mathcal{O}_{V}$-free of rank $n-1$ and we have $\Theta(-\log D)=\Theta(-\log h) \oplus \mathcal{O}_{V} \cdot E$, where $E=\sum_{i=1}^{n} x_{i} \partial_{x_{i}}$.

2. $D$ is called reductive, if the identity component $G$ of the algebraic group $G_{D}:=\{g \in \operatorname{Gl}(V) \mid g(D) \subset D\}$ is so.

3. For any reductive linear free divisor $D$, the dual action of $G$ on $V^{\vee}$ has an open orbit, with complement a reduced hypersurface $D^{\vee}$. Any linear form $f$ in the open orbit $V^{\vee} \backslash D^{\vee}$ is called generic.

We are interested in Gauß-Manin systems of the restriction of generic linear forms to the Milnor fibres of $D$, this leads to consider relative de Rham complexes with twisted differentials.

Definition-Lemma 2. Let $D \subset V$ be a reductive linear free divisor with defining equation $h$, seen as a morphism $h: V \rightarrow T=\operatorname{Spec} \mathbb{C}[t]$. Moreover, let $f \in V^{\vee} \backslash D^{\vee}$ be a generic linear form, which we see as a morphism $f: V \rightarrow S=$ Spec $\mathbb{C}[s]$.

Put

$$
\begin{aligned}
G(\log D):=\mathbb{H}^{n}\left(\Omega_{V / T}^{\bullet}(\log D)\left[\theta, \theta^{-1}\right], \theta d-d f \wedge\right) & ; \quad G(* D):=\mathbb{H}^{n-1}\left(\Omega_{V / T}^{\bullet}(* D)\left[\theta, \theta^{-1}\right], \theta d-d f \wedge\right) \\
G_{0}(\log D):=\mathbb{H}^{n}\left(\Omega_{V / T}^{\bullet}(\log D)[\theta], \theta d-d f \wedge\right) & ; \quad G_{0}(* D):=\mathbb{H}^{n-1}\left(\Omega_{V / T}^{\bullet}(* D)[\theta], \theta d-d f \wedge\right)
\end{aligned}
$$

where $\Omega_{V / T}^{\bullet}(* D):=\Omega_{V}^{\bullet}(* D) / h^{*} \Omega_{T}^{1}(*\{0\}) \wedge \Omega_{V}^{\bullet-1}(* D)$ resp. $\Omega_{V / T}^{\bullet}(\log D):=\Omega_{V}^{\bullet}(\log D) / h^{*} \Omega_{T}^{1}(\log \{0\}) \wedge \Omega_{V}^{\bullet-1}(\log D)$ is the localization along $D$ resp. its logarithmic extension over $D$ of the relative de Rham complex of $h$. Then $G(* D)$ is $\mathbb{C}\left[\theta, \theta^{-1}, t, t^{-1}\right]$-free of rank $n$ and $G(\log D)$ (resp. $G_{0}(* D), G_{0}(\log D)$ ) is a $\mathbb{C}\left[\theta, \theta^{-1}, t\right]-\left(\right.$ resp. $\mathbb{C}\left[\theta, t, t^{-1}\right]$-, $\mathbb{C}[\theta, t]-$ ) lattice inside $G(* D)$. There is a connection operator

$$
\nabla: G_{0}(\log D) \longrightarrow G_{0}(\log D) \otimes z^{-1} \Omega_{\mathbb{C} \times T}^{1}(\log ((\{0\} \times T) \cup(\mathbb{C} \times\{0\})))
$$

which induces connections on $G(\log D), G_{0}(* D)$ and $G(* D)$.

We have constructed in [GMS09] two particular bases of $G_{0}(* D)$ in which the connection can be expressed in a very simple way. This is summarized in the following proposition.

Proposition 3. 1. There is a $\mathbb{C}\left[\theta, t, t^{-1}\right]$-basis $\underline{\omega}=\left(\omega_{1}, \ldots, \omega_{n}\right)$ (called $\underline{\omega}^{(2)}$ in [GMS09, corollary 4.12]) of $G_{0}(* D)$ such that

$$
\nabla(\underline{\omega})=\underline{\omega} \cdot\left[\left(A_{0} \frac{1}{\theta}+A_{\infty}\right) \frac{d \theta}{\theta}+\left(-A_{0} \frac{1}{\theta}+A_{\infty}^{\prime}\right) \frac{d t}{n t}\right]
$$

where

$$
A_{0}:=\left(\begin{array}{ccccc}
0 & 0 & \ldots & 0 & c \cdot t \\
-1 & 0 & \ldots & 0 & 0 \\
\vdots & \vdots & \ddots & \vdots & \vdots \\
0 & 0 & \ldots & 0 & 0 \\
0 & 0 & \ldots & -1 & 0
\end{array}\right),
$$

$c \in \mathbb{C}^{*}, A_{\infty}=\operatorname{diag}\left(\nu_{1}, \ldots, \nu_{n}\right)$ and $A_{\infty}^{\prime}:=\operatorname{diag}(0,1, \ldots, n-1)-A_{\infty}$. The numbers $\nu_{i}$ have the following two properties:

(a) For all $i \in\{1, \ldots, n-1\}$, we have $\nu_{i+1}-\nu_{i} \leq 1$.

(b) Let $\sigma \in S_{n}$ be a permutation such that $\nu_{\sigma(1)} \leq \ldots \leq \nu_{\sigma(n)}$. Then we have the symmetry $\nu_{\sigma(i)}+\nu_{\sigma(n+1-i)}=$ $n-1$.

We have $G_{0}(\log D)=\oplus_{i=1}^{n} \mathbb{C}[t, \theta] \omega_{i}$ and the basis $\underline{\omega}$ is a $V^{+}$-solution to the Birkhoff problem (see [DS03, appendix B.d]) of the module $\left(G_{0}(\log D) / t \cdot G_{0}(\log D), \nabla\right)$, which was called logarithmic Brieskorn lattice in [Sev11].

2. There is another basis $\underline{\widetilde{\omega}}$ of $G_{0}(* D)$ (called $\underline{\omega}^{(3)}$ in [GMS09, corollary 4.12]) for which the connection matrix takes the same form as for $\underline{\omega}$, that is

$$
\nabla(\underline{\widetilde{\omega}})=\underline{\widetilde{\omega}} \cdot\left[\left(A_{0} \frac{1}{\theta}+\widetilde{A}_{\infty}\right) \frac{d \theta}{\theta}+\left(-A_{0} \frac{1}{\theta}+\widetilde{A}_{\infty}^{\prime}\right) \frac{d t}{n t}\right]
$$


where $\widetilde{A}_{\infty}=\operatorname{diag}\left(\widetilde{\nu}_{1}, \ldots, \widetilde{\nu}_{n}\right)$ and $\widetilde{A}_{\infty}^{\prime}=\operatorname{diag}(0,1, \ldots, n-1)-\widetilde{A}_{\infty}$. Here the numbers $\widetilde{\nu}_{i}$ have the same properties as the numbers $\nu_{i}$ above and satisfy additionally $\widetilde{\nu}_{1}-\widetilde{\nu}_{n} \leq 1$, moreover, we have $\underline{\omega}=\underline{\widetilde{\widetilde{\omega}}}$ if $\nu_{1}-\nu_{n} \leq 1$. $\underline{\widetilde{\omega}}$ is a $V^{+}$-solution to the Birkhoff problem in a family for $\left(G_{0}(* D), \nabla\right)$.

The following theorem is a rather easy consequence of this result, but it will be very useful when studying the duality theory of $G(* D)$.

Theorem 4. Write $\mathcal{D}=\mathbb{C}\left[\theta, \theta^{-1}, t, t^{-1}\right]\left\langle\partial_{t}, \partial_{\theta}\right\rangle$. Then there is an isomorphism of left $\mathcal{D}$-modules

$$
\begin{aligned}
\varphi: \mathcal{G}:=\mathcal{D} / \mathcal{D} \cdot\left(P_{1}, P_{2}\right) & \longrightarrow G(* D) \\
1 & \longmapsto[n \mathrm{vol} / d h],
\end{aligned}
$$

where

$$
P_{1}:=\prod_{i=1}^{n} \theta\left(t \partial_{t}-\frac{i-1-\nu_{i}}{n}\right)-\frac{c}{n^{n}} \cdot t \quad ; \quad P_{2}:=\theta^{2} \partial_{\theta}+n \cdot t \theta \partial_{t}
$$

and where $\mathrm{vol}=d x_{1} \wedge \ldots \wedge d x_{n}$. The inverse image of the lattice $G_{0}(* D)$ under $\varphi$ can be described as the subring $\mathcal{G}_{0}$ of $\mathcal{D} / \mathcal{D} \cdot\left(P_{1}, P_{2}\right)$ defined as

$$
\mathcal{G}_{0}:=\mathbb{C}\left[\theta, t, t^{-1}\right]\left\langle\theta \partial_{t}, \theta^{2} \partial_{\theta}\right\rangle / \mathbb{C}\left[\theta, t, t^{-1}\right]\left\langle\theta \partial_{t}, \theta^{2} \partial_{\theta}\right\rangle \cdot\left(P_{1}, P_{2}\right) .
$$

Here $\mathbb{C}\left[\theta, t, t^{-1}\right]\left\langle\theta \partial_{t}, \theta^{2} \partial_{\theta}\right\rangle$ is the $\mathbb{C}\left[\theta, t, t^{-1}\right]$-subalgebra of $\mathcal{D}$ generated by $\theta \partial_{t}$ and $\theta^{2} \partial_{\theta}$.

Proof. By forgetting the $\partial_{\theta}$-action, we can see $G(* D)$ as a $\mathbb{C}\left[\theta, \theta^{-1}, t, t^{-1}\right]\left\langle\partial_{t}\right\rangle$-module only. The first step is then to show that there is an isomorphism of $\mathbb{C}\left[\theta, \theta^{-1}, t, t^{-1}\right]\left\langle\partial_{t}\right\rangle$-modules

$$
\begin{aligned}
\widetilde{\varphi}: \widetilde{\mathcal{G}}:=\mathbb{C}\left[\theta, \theta^{-1}, t, t^{-1}\right]\left\langle\partial_{t}\right\rangle / \mathbb{C}\left[\theta, \theta^{-1}, t, t^{-1}\right]\left\langle\partial_{t}\right\rangle \cdot P_{1} & \longrightarrow G(* D) \\
1 & \longmapsto[n \mathrm{vol} / d h],
\end{aligned}
$$

From the form of the operator $P_{1}$ we see that the left hand side is generated over $\mathbb{C}\left[\theta, \theta^{-1}, t, t^{-1}\right]$ by the elements $\left(Q_{i}:=\prod_{j=1}^{i} \theta\left(t \partial_{t}-\frac{j-1-\nu_{j}}{n}\right)\right)_{i=0, \ldots, n-1}$, where we put by definition $Q_{0}:=1$. Hence the operator $\theta t \partial_{t}-\frac{i-1-\nu_{i}}{n}$ sends $Q_{i-1}$ to $Q_{i}$, so that by setting $\widetilde{\varphi}\left(Q_{i}\right):=n^{-i} \omega_{i+1}$, we obtain a $\mathbb{C}\left[\theta, \theta^{-1}, t, t^{-1}\right]$-linear map which is compatible with the action of $\partial_{t}$ on the left hand side and $\nabla_{\partial_{t}}$ on the right hand side. Due to the particular form of the operator $P_{2}$ (more precisely, due to the fact that $\left.\theta^{-2} \cdot P_{2}=\partial_{\theta}+n \theta^{-1} t \partial_{t} \in\left(P_{1}, P_{2}\right)\right)$, the module $\mathcal{G}$ is isomorphic to $\widetilde{\mathcal{G}}$ when seen as a $\mathbb{C}\left[\theta, \theta^{-1}, t, t^{-1}\right]\left\langle\partial_{t}\right\rangle$-module only. Hence in order to finish the proof of the first statement, we have to check that the action of $\partial_{\theta}$ on $\mathcal{G}$ coincides with the action of $\nabla_{\partial_{\theta}}$ on $G(* D)$, which is clear from formula (1), and by noticing that

$$
\begin{aligned}
\left(\theta^{2} \partial_{\theta}-\theta \nu_{i-1}\right) \cdot Q_{i-1} & =(i-1) \theta^{i} \cdot R_{i-1}-\theta^{i-1} \cdot R_{i-1} \cdot n \theta t \partial_{t}-\theta^{i} \nu_{i} \cdot R_{i-1} \\
\theta^{i} \cdot\left(-n t \partial_{t}+i-1-\nu_{i}\right) \cdot R_{i-1} & =-n \cdot Q_{i},
\end{aligned}
$$

where we write $R_{i}=\prod_{j=1}^{i}\left(t \partial_{t}-\frac{j-1-\nu_{j}}{n}\right)$.

Looking at the connection matrix $(1)$, one immediately sees that $\varphi\left(\mathcal{G}_{0}\right) \subset G_{0}(* D)$. In order to show equality, take any section $[\omega] \in G_{0}(* D)$ with representative $\omega=\sum_{k \geq 0} \theta^{k} \omega^{(k)}$, where $\omega^{(k)} \in \Omega_{V / T}^{n-1}(* D)$. There is an (uniquely determined) operator $P$ in $\mathcal{G}$ with $\varphi(P)=[\omega]$, and we have to show that $P \in \mathcal{G}_{0}$. By linearity of $\varphi$, it is sufficient to do it for the case where $\omega^{(0)} \neq 0$, and then there is a minimal $k \in \mathbb{N}$ with $\theta^{k} \cdot P \in \mathcal{G}_{0}$, and then the class of $\theta^{k} \cdot P$ in $\mathcal{G}_{0} / \theta \mathcal{G}_{0}$ is non-zero. On the other hand, $\theta^{k} \varphi(P)=\theta^{k} \omega \in G_{0}(* D)$, and the class of $\theta^{k} \omega$ is zero in $G_{0}(* D) / \theta \cdot G_{0}(* D)$ unless $k=0$. Hence the statement follows once we know that the induced morphism

$$
[\widetilde{\varphi}]: \mathcal{G}_{0} / \theta \mathcal{G}_{0} \longrightarrow G_{0}(* D) / \theta G_{0}(* D)
$$

is an isomorphism. Now recall from [GMS09, section 3.2] that the relative deformation or Jacobian algebra is defined as

$$
\mathcal{T}_{\mathcal{R}_{h} / T}^{1}(f):=\frac{\mathcal{O}_{V}}{d f(\Theta(-\log h))} .
$$

Then we have $G_{0}(* D) / \theta G_{0}(* D) \cong H^{0}\left(V, \mathcal{T}_{\mathcal{R}_{h} / T}^{1}(f)\right) \cdot$ vol. On the other hand, $\mathcal{G}_{0} / \theta \mathcal{G}_{0} \cong \widetilde{\mathcal{G}}_{0} / \theta \widetilde{\mathcal{G}}_{0}=\mathbb{C}\left[t, t^{-1}, \mu\right] /\left((t \mu)^{n}-\right.$ $\left.c / n^{n} \cdot t\right)$, where we denote by $\mu$ the class of $\left[\theta \partial_{t}\right]$ in $\widetilde{\mathcal{G}}_{0} / \theta \widetilde{\mathcal{G}}_{0}$. Then the isomorphism $\mathbb{C}\left[t, t^{-1}, \mu\right] /\left((t \mu)^{n}-c / n^{n} \cdot t\right) \cong$ $H^{0}\left(V, \mathcal{T}_{\mathcal{R}_{h} / T}^{1}(f)\right)$ follows from [GMS09, proposition 3.5]. 


\section{Duality theorems}

In this sections we derive the existence of a pairing on the meromorphic bundle $G(* D)$. For that purpose, we compute the holonomic dual of the module $\mathcal{G}$. We show that it is self-dual, by exhibiting a $\mathcal{D}$-free resolution of it. Using theorem 4 and a comparison result between the meromorphic and the holonomic dual module of a meromorphic bundle, this yields the pairing mentioned above. We also show that it is compatible with the lattice $G_{0}(* D)$, this fact is used later for the construction of the flat metric on the Frobenius manifold associated to the pair $(f, h)$.

Proposition 5. 1. Let $\mathcal{G}^{r}:=\mathcal{D} /\left(P_{1}^{t},-P_{2}^{t}\right) \cdot \mathcal{D}$ be the right module associated to $\mathcal{G}$, here

$$
P_{1}^{t}:=\prod_{i=1}^{n}(-\theta)\left(t \partial_{t}+\frac{i-1-\nu_{i}}{n}+1\right)-\frac{c}{n^{n}} \cdot t \quad ; \quad P_{2}^{t}:=-\left(\theta^{2} \partial_{\theta}+n \cdot t \theta \partial_{t}+\theta(n+2)\right) .
$$

are the usual transforms of $P_{1}$ and $P_{2}$. Then $\mathcal{G}^{r}$ has a the following explicit resolution by free right $\mathcal{D}$-modules

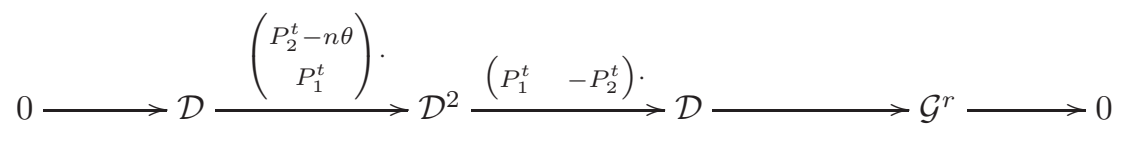

2. We have $\mathbb{D} \mathcal{G}=\mathcal{D} / \mathcal{D} \cdot\left(\widetilde{P}_{1}, \widetilde{P}_{2}\right)$ where $\widetilde{P}_{1}=P_{1}^{t}$ and $\widetilde{P}_{2}:=-P_{2}^{t}+n \theta=\theta^{2} \partial_{\theta}+n t \theta \partial_{t}+\theta(2 n+2)$. Moreover, there is an isomorphism $\Phi: \mathcal{G} \rightarrow \iota^{*} \mathbb{D} \mathcal{G}$ of left $\mathcal{D}$-modules, induced by $\Phi(m)=m \cdot \theta^{n+2} \cdot t$ for any $m \in \mathcal{G}$, where $\iota$ is the involution sending $z$ to $-z$.

Proof. 1. From the commutation relation $\left[P_{1}^{t}, P_{2}^{t}\right]=n \cdot \theta \cdot P_{1}^{t}$ we conclude that $(3)$ is a complex. In order to show that it is exact, filter $\mathcal{D}$ as usual by orders of operators. Then it suffices to show that the graded object with respect to this filtration of the complex (3) is acyclic. It is easy to see that the symbols $\sigma\left(P_{1}^{t}\right)=\left(-\theta t \sigma\left(\partial_{t}\right)\right)^{n}$ and $\sigma\left(P_{2}^{t}\right)=-\theta^{2} \sigma\left(\partial_{\theta}\right)-n t \theta \sigma\left(\partial_{t}\right)$ form a regular sequence in $\operatorname{gr}(\mathcal{D})$, so that the only relation between them is the Koszul relation, and the corresponding (exact) Koszul complex is exactly the graded complex associated to the above complex of $\mathcal{D}$-modules, which is hence acyclic.

2. As the above sequence (3) is a free right resolution, we obtain that $\mathbb{D} \mathcal{G}$ is the top cohomology group of its dual complex (which is naturally a complex of left $\mathcal{D}$-modules, notice that we could have as well started with a left resolution of $\mathcal{G}$, use it to compute the right $\mathcal{D}$-module $\operatorname{Ext}_{\mathcal{D}}^{2}(\mathcal{G}, \mathcal{D})$ and then obtain $\mathbb{D} \mathcal{G}$ as the left transform of it). It follows from [Sev11, corollary 17] that $\widetilde{P}_{1}=\prod_{i=1}^{n}(-\theta)\left(t \partial_{t}-\frac{i-1-\nu_{i}}{n}+1\right)-\frac{c}{n^{n}} \cdot t$, due to the symmetry of the numbers $\frac{i-1-\nu_{i}}{n}$ deduced in loc.cit. from the symmetry of the roots of the Bernstein polynomial $b_{h}$ shown in [GS10]. Now one checks explicitly that the morphism

$$
\begin{aligned}
\Phi: \mathcal{D} / \mathcal{D} \cdot\left(P_{1}, P_{2}\right) & \longrightarrow \iota^{*}\left(\mathcal{D} / \mathcal{D} \cdot\left(\widetilde{P}_{1}, \widetilde{P}_{2}\right)\right) \\
m & \longmapsto m \cdot \theta^{n+2} \cdot t
\end{aligned}
$$

is well-defined, i.e., that $P_{1}\left(\theta^{n+2} t\right)=P_{2}\left(\theta^{n+2} t\right)=0 \in \iota^{*}\left(\mathcal{D} / \mathcal{D}\left(\widetilde{P}_{1}, \widetilde{P}_{2}\right)\right)$. It is obviously invertible, and hence yields the desired isomorphism $\Phi: \mathcal{G} \rightarrow \iota^{*} \mathbb{D} \mathcal{G}$.

From the above calculation we can now deduce the first main result.

Theorem 6. There is a non-degenerate, $(-1)^{n-1}$-symmetric pairing $S: G(* D) \otimes \iota^{*} G(* D) \rightarrow \mathbb{C}\left[\theta, \theta^{-1}, t, t^{-1}\right]$, which is compatible with the connections.

Proof. We first recall a construction from [DS03, lemma A.11] and [Sai89, section 2.7] which yields a canonical resolution of the right $\mathcal{D}$-module associated to $G(* D)$ and, as a consequence, an identification of the holonomic and the meromorphic dual of $G(* D)$. We will write $\mathcal{O}:=\mathcal{O}_{\widehat{S}^{*} \times T^{*}}$ and $\Omega^{i}:=\Omega_{\widehat{S}^{*} \times T^{*}}^{i}$, with $\widehat{S}^{*}=\operatorname{Spec} \mathbb{C}\left[\theta, \theta^{-1}\right]$.

Consider the de Rham complex $\Omega^{\bullet}(\mathcal{D})$ of $\mathcal{D}$ which is a resolution by free right $\mathcal{D}$-modules of $\Omega^{2}$, i.e., the exact sequence

$$
0 \longrightarrow \mathcal{D} \stackrel{\alpha^{\prime}}{\longrightarrow} \Omega^{1} \otimes_{\mathcal{O}} \mathcal{D} \stackrel{\beta^{\prime}}{\longrightarrow} \Omega^{2} \otimes_{\mathcal{O}} \mathcal{D} \stackrel{\gamma^{\prime}}{\longrightarrow} \Omega^{2} \longrightarrow 0,
$$

where $\alpha^{\prime}(P)=d \theta \otimes\left(\partial_{\theta} \cdot P\right)+d t \otimes\left(\partial_{t} \cdot P\right), \beta^{\prime}\left(d \theta \otimes P_{1}+d t \otimes P_{2}\right)=(d \theta \wedge d t) \otimes\left(\partial_{\theta} \cdot P_{2}-\partial_{t} \cdot P_{1}\right)$ and $\gamma^{\prime}((d \theta \wedge d t) \otimes Q)=$ $(d \theta \wedge d t) \cdot Q$, where the last term denotes the result of the right action of the operator $Q$ on the element $d \theta \wedge d t \in \Omega^{2}$. 
Now recall that there is a sequence of isomorphism of right $\mathcal{D}$-modules

$$
\left(\Omega^{i} \otimes \mathcal{D}\right) \otimes G(* D) \cong \Omega^{i} \otimes(\mathcal{D} \otimes G(* D)) \cong \Omega^{i} \otimes(G(* D) \otimes \mathcal{D}) \cong\left(\Omega^{i} \otimes G(* D)\right) \otimes \mathcal{D}
$$

where all tensor products are over $\mathcal{O}$, where the left-most and the right-most isomorphisms are the obvious ones, and where the middle-one is induced by the isomorphism $\mathcal{D} \otimes G(* D) \stackrel{\cong}{\rightrightarrows} G(* D) \otimes \mathcal{D}$ sending $P \otimes m$ to $P \cdot(m \otimes 1)$. Notice that here $G(* D) \otimes \mathcal{D}$ carries the trivial right $\mathcal{D}$-module structure (i.e., the one coming from right multiplication on the second factor), but also the left $\mathcal{D}$-module structure induced by the action $g(m \otimes P)=m \otimes g P$ for $g \in \mathcal{O}$ and $\xi(m \otimes P):=\xi m \otimes P+m \otimes \xi P$ for $\xi \in \operatorname{Der}(\mathcal{O}, \mathcal{O}) \subset \mathcal{D}$. Similarly, $\mathcal{D} \otimes G(* D)$ has the trivial left structure, but also a (non-trivial) right structure defined similarly to the left structure of $G(* D) \otimes \mathcal{D}$. Using these isomorphisms, one checks that the right $\mathcal{D}$-module complex $\Omega^{\bullet}(\mathcal{D}) \otimes G(* D)$ is isomorphic to

$$
0 \longrightarrow G(* D) \otimes_{\mathcal{O}} \mathcal{D} \stackrel{\alpha}{\longrightarrow} \Omega^{1} \otimes_{\mathcal{O}} G(* D) \otimes_{\mathcal{O}} \mathcal{D} \stackrel{\beta}{\longrightarrow} \Omega^{2} \otimes_{\mathcal{O}} G(* D) \otimes_{\mathcal{O}} \mathcal{D} \stackrel{\gamma}{\longrightarrow} \Omega^{2} \otimes_{\mathcal{O}} G(* D) \longrightarrow 0
$$

where $\alpha$ (resp. $\beta$ and $\gamma$ ) are induced from $\alpha^{\prime} \otimes I d_{G(* D)}\left(\right.$ resp. $\beta^{\prime} \otimes I d_{G(* D)}$ and $\left.\gamma^{\prime} \otimes I d_{G(* D)}\right)$ under the isomorphisms (4) and can be expressed explicitly as follows

$$
\begin{aligned}
\alpha(m \otimes P) & =d \theta \otimes\left(\nabla_{\theta} m \otimes P+m \otimes \partial_{\theta} P\right)+d t \otimes\left(\nabla_{t} m \otimes P+m \otimes \partial_{t} P\right) \\
\beta\left(d \theta \otimes m_{1} \otimes P_{1}+d t \otimes m_{2} \otimes P_{2}\right) & =d \theta \wedge d t \otimes\left(\nabla_{\theta} m_{2} \otimes P_{2}+m_{2} \otimes \partial_{\theta} P_{2}-\nabla_{t} m_{1} \otimes P_{1}-m_{1} \otimes \partial_{t} P_{1}\right) \\
\gamma(d \theta \wedge d t \otimes(m \otimes P)) & =((d \theta \wedge d t) \cdot P) \otimes m-(d \theta \wedge d t) \otimes(P \cdot m)
\end{aligned}
$$

where, as before $(d \theta \wedge d t) \cdot P$ denotes the right action of the operator $P \in \mathcal{D}$ on the form $d \theta \wedge d t \in \Omega^{2}$ and $P \cdot m$ denotes the left action of $P$ on $m$ using the connection on the meromorphic bundle $G(* D)$. Notice that as $G(* D)$ is $\mathcal{O}$-free, the complex (5) is still exact, in other words, it yields a canonical resolution by free right $\mathcal{D}$-modules of the right module associated to $G(* D)$.

The isomorphism $\varphi: \mathcal{G} \rightarrow G(* D)$ from theorem 4 induces an isomorphism $\varphi^{r}$ on the associated right $\mathcal{D}$-modules, and the latter extends to an isomorphism of complexes

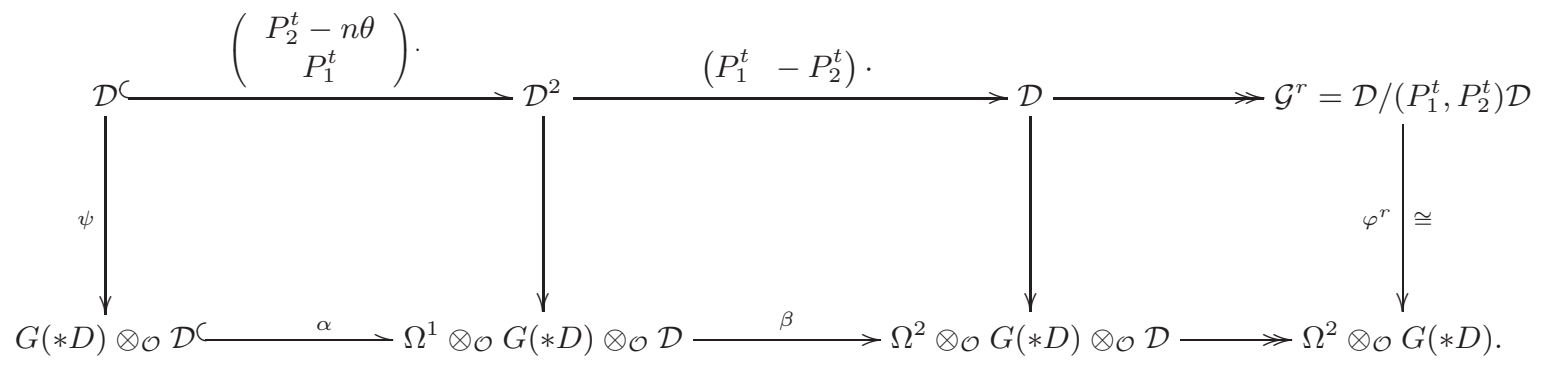

Applying the functor $\mathcal{H o m}_{\mathcal{D}}(-, \mathcal{D})$ to the free part of the above diagram (i.e., to the morphism between the free resolutions of $\mathcal{G}^{r}$ resp. $\left.\Omega^{2} \otimes G(* D)\right)$ and using the isomorphism $\mathcal{H}_{0} m_{\mathcal{D}}(\mathcal{F} \otimes \mathcal{D}, \mathcal{D})=\mathcal{H}_{0} m_{\mathcal{O}}(\mathcal{F}, \mathcal{D}) \cong \mathcal{D} \otimes \mathcal{F}^{\vee}$ for any $\mathcal{O}$-free module $\mathcal{F}$, we see that the free resolution of $\Omega^{2} \otimes G(* D)$ is transformed to the complex $G(* D)^{\vee} \otimes S p^{\bullet}(\mathcal{D})$, where $S p^{\bullet}(\mathcal{D})$ denotes the Spencer complex of $\mathcal{D}$, i.e., a resolution of $\mathcal{D}$ by free left $\mathcal{D}$-modules. This is a free left resolution of the left $\mathcal{D}$-modules $G(* D)^{\vee}$. Hence the transpose of $\psi$ induces an isomorphism $\Psi: G(* D)^{\vee} \rightarrow \mathbb{D} \mathcal{G}$ of left $\mathcal{D}$-modules.

The existence of the pairing $S$ can be rephrased as an isomorphism $\Phi^{m e r}:(G(* D), \nabla) \stackrel{\cong}{\rightrightarrows} \iota^{*}(G(* D), \nabla)^{\vee}$ of meromorphic bundles with connection (here $(G(* D), \nabla)^{\vee}$ denotes the dual vector bundle together with its dual connection). We define $\Phi^{\text {mer }}$ by the commutative diagram

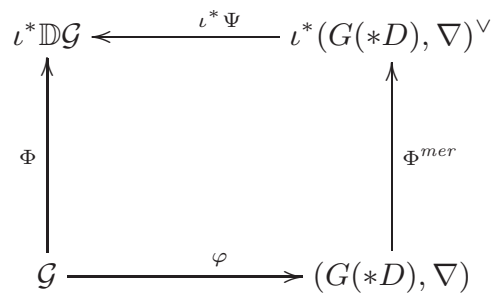


where $\Phi$ is the morphism from proposition 5,2 . Notice that $\Phi^{m e r}$ is an isomorphism as $\Phi, \varphi$ and $\iota^{*} \Psi$ are so. In order to show the $(-1)^{n-1}$-symmetry of $S$, we use a variant of a criterion from [DS03, corollary 1.23]. Namely, it is sufficient to show that the morphism

$$
\iota^{*} \mathbb{D} \Phi: \iota^{*} \mathbb{D}\left(\iota^{*} \mathbb{D} \mathcal{G}\right)=\mathcal{G} \longrightarrow \iota^{*} \mathbb{D} \mathcal{G}
$$

satisfies $\iota^{*} \mathbb{D} \Phi=(-1)^{n-1} \cdot \Phi$. This can be proved by computing a resolution of the right module $\iota^{*}(\mathbb{D} \mathcal{G})^{r}=\iota^{*} \mathcal{E} x t_{\mathcal{D}}(\mathcal{G}, \mathcal{D})$, extending the morphism $\Phi^{r}: \mathcal{G}^{r} \rightarrow\left(\iota^{*} \mathbb{D} \mathcal{G}\right)^{r}$ to a morphism of the corresponding resolutions and dualizing. In other words, we consider the following morphism of complexes

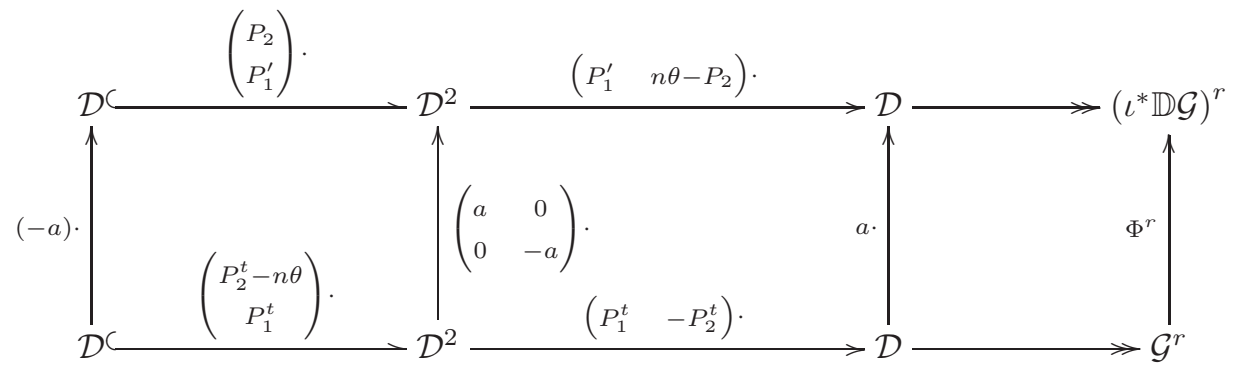

where $P_{1}^{\prime}=\prod_{i=1}^{n}(-\theta)\left(t \partial_{t}-\frac{i-1-\nu_{i}}{n}\right)-\frac{c}{n^{n}} \cdot t$ and where we have put $a:=\theta^{n+2} t$ for short. The dual of the leftmost morphism induces the map

$$
\begin{aligned}
\mathbb{D} \Phi: \mathbb{D} \iota^{*} \mathbb{D} \mathcal{G} \cong \iota^{*} \mathcal{G} \cong \mathcal{D} / \mathcal{D}\left(P_{1}^{\prime}, P_{2}\right) & \longrightarrow \mathbb{D} \mathcal{G}=\mathcal{D} / \mathcal{D}\left(P_{1}^{t}, n \theta-P_{2}^{t}\right) \\
m & \longmapsto m \cdot(-a)=m \cdot(-1) \cdot \theta^{n+2} t
\end{aligned}
$$

Therefore the morphism $\iota^{*} \mathbb{D} \Phi$ is given by right multiplication with $(-1) \cdot(-\theta)^{n+2} t$ and hence satisfies $\iota^{*} \mathbb{D} \Phi=$ $(-1)^{n+1} \cdot \Phi=(-1)^{n-1} \cdot \Phi$, as required.

In the remainder of this section, we will show a more refined version of theorem 6 taking into account the behavior of the pairing $S$ with respect to the lattice $G_{0}(* D)$. More precisely, we have the following result.

Theorem 7. The pairing $S$ from theorem 6 satisfies $S\left(G_{0}(* D), G_{0}(* D)\right) \subset \theta^{n-1} \mathbb{C}\left[\theta, \theta^{-1}, t, t^{-1}\right]$. Moreover, it induces a non-degenerate symmetric pairing $S_{0}:\left(G_{0}(* D) / \theta \cdot G_{0}(* D)\right) \otimes\left(G_{0}(* D) / \theta \cdot G_{0}(* D)\right) \rightarrow \theta^{n-1} \mathbb{C}\left[t, t^{-1}\right]$.

Proof. It is clear that the statement of the theorem is equivalent to the fact that the morphism $\Phi^{m e r}: G(* D) \stackrel{\cong}{\longrightarrow}$ $\iota^{*} G(* D)$ appearing in the proof of the previous theorem sends $G_{0}(* D)$ isomorphically onto $\iota^{*}\left(\theta^{n-1} \cdot G_{0}(* D)^{\vee}\right)$, where

$$
G_{0}(* D)^{\vee}=\mathcal{H o m}_{\mathbb{C}\left[\theta, t, t^{-1}\right]}\left(G_{0}(* D), \mathbb{C}\left[\theta, t, t^{-1}\right]\right) \cong\left\{l \in G(* D)^{\vee} \mid l\left(G_{0}(* D)\right) \subset \mathbb{C}\left[\theta, t, t^{-1}\right]\right\} \subset G(* D)^{\vee} .
$$

In order to show this statement, we will consider completions along $\theta=0$. We write $\mathcal{O}^{\wedge}:=\mathbb{C}[[\theta]]\left[\theta^{-1}, t, t^{-1}\right]$. For any $\mathcal{O}$-module $\mathcal{F}$ we denote by $\mathcal{F}^{\wedge}$ the tensor product with $\mathcal{O}^{\wedge}$. We have thus a duality isomorphism $\Phi^{m e r, \wedge}: G(* D)^{\wedge} \longrightarrow$ $\left(\iota^{*} G(* D)^{\vee}\right)^{\wedge}$. As both $\iota^{*}\left(\theta^{n-1} \cdot G_{0}(* D)^{\vee}\right)$ and $\Phi^{m e r}\left(G_{0}(* D)\right)$ are lattices inside $\iota^{*}\left(G(* D)^{\vee}\right)$, it is sufficient to show that $\Phi^{m e r, \wedge}: G_{0}(* D)^{\wedge} \cong\left(\iota^{*}\left(\theta^{n-1} \cdot G_{0}(* D)^{\vee}\right)\right)^{\wedge}$. In order to show this property, we will consider the formal versions of the exact sequences $(3)$ and $(5)$, which are exact sequences of $\mathcal{D}^{\wedge}:=\mathbb{C}[[\theta]]\left[\theta^{-1}, t, t^{-1}\right]\left\langle\partial_{\theta}, \partial_{t}\right\rangle$-modules.

We have thus the following formal version of the diagram (6)

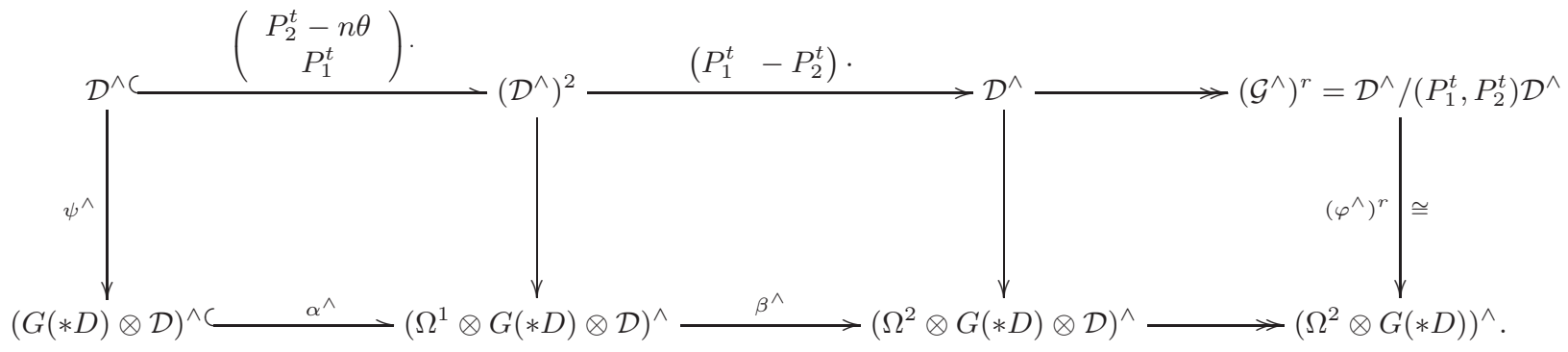


Consider as in [DS03, lemma A.12] the filtration $F_{\bullet}$ on $\mathcal{D}^{\wedge}$ which extends the order filtration on $\mathbb{C}\left[t, t^{-1}\right]\left\langle\partial_{t}\right\rangle$ and for which $\partial_{\theta}$ has degree two and $\theta$ has degree -1 (this is not the usual filtration by order as considered in the proof of proposition 5, 1. above). Notice that by an argument like in [SST00, page 4], we see that $g r_{.}^{F} \mathcal{D}^{\wedge} \cong$ $\mathbb{C}[[\theta]]\left[\theta^{-1}, t, t^{-1}, u, v\right]$, where $u$ resp. $v$ represents the class of $\partial_{\theta}$ resp. $\partial_{t}$. The ring $g r_{\bullet}^{F}$ is graded by $\operatorname{deg}(t)=0$, $\operatorname{deg}(\theta)=-1, \operatorname{deg}(u)=2$ and $\operatorname{deg}(v)=1$. Notice further that $F_{\bullet}$ induces a good filtration on $\mathcal{G}^{\wedge}$ with $\mathcal{G}_{0}^{\wedge}=F_{0} \mathcal{G}^{\wedge}$, and that we have $F_{k} \mathcal{G}^{\wedge}=\theta^{-k} \mathcal{G}_{0}^{\wedge}$, this follows from the fact that $\mathcal{G}^{\wedge}=\oplus_{i=0}^{n-1} \mathbb{C}[[\theta]]\left[\theta^{-1}, t, t^{-1}\right] Q_{i}$ and that any $Q_{i}$ is of degree zero (i.e. the minimal $k$ such that $Q_{i} \in F_{k} \mathcal{D}^{\wedge}$ is zero). Moreover, put $F_{k} G(* D)^{\wedge}:=\theta^{-k} G_{0}(* D)^{\wedge}$, then as $F_{k} \mathcal{G}^{\wedge}=\theta^{-k} \mathcal{G}_{0}^{\wedge}$ the isomorphism $\varphi^{\wedge}: \mathcal{G}^{\wedge} \rightarrow G(* D)^{\wedge}$ induced from the isomorphism $\varphi$ from theorem 4 is strictly filtered.

We will consider induced filtrations $F_{\bullet}\left(\Omega^{1}\right)^{\wedge}$ resp. $F_{\bullet}\left(\Omega^{2}\right)^{\wedge}$ defined by the filtration on $\mathcal{O}^{\wedge}$ induced from the filtration on $\mathcal{D}^{\wedge}$. These filtrations are defined such that $d \theta \in F_{-2}\left(\Omega^{1}\right)^{\wedge} \backslash F_{-3}\left(\Omega^{1}\right)^{\wedge}, d t \in F_{-1}\left(\Omega^{1}\right)^{\wedge} \backslash F_{-2}\left(\Omega^{1}\right)^{\wedge}$ and $d \theta \wedge d t \in$ $F_{-3}\left(\Omega^{2}\right)^{\wedge} \backslash F_{-4}\left(\Omega^{2}\right)^{\wedge}$. The map of right $\mathcal{D}^{\wedge}$-modules $\left(\varphi^{\wedge}\right)^{r}:\left(\mathcal{G}^{\wedge}\right)^{r} \rightarrow\left(\left(\Omega^{2}\right) \otimes G(* D)\right)^{\wedge}$ associated to the morphism $\varphi^{\wedge}$ sends 1 to $(d \theta \wedge d t) \otimes(\operatorname{vol} / d h)$ and thus satisfies $\left(\varphi^{\wedge}\right)^{r}: F_{\bullet}\left(\mathcal{G}^{\wedge}\right)^{r} \stackrel{\cong}{\rightarrow} F_{\bullet-3}\left(\Omega^{2} \otimes G(* D)\right)^{\wedge}$.

Notice that we have $\nabla_{\theta} F_{k} G(* D)^{\wedge} \subset F_{k+2} G(* D)^{\wedge}$ (because $G_{0}(* D)$ is stable by $\theta^{2} \nabla_{\theta}$ ) and that similarly the inclusion $\nabla_{t} F_{k} G(* D)^{\wedge} \subset F_{k+1} G(* D)^{\wedge}$ holds. Using our convention for the induced filtration on $\Omega^{1}$ and $\Omega^{2}$, this shows that the formal version of the resolution (5) is filtered. The same is obviously true for the formal version of the sequence (3), as all components of the matrices defining the differentials in that sequence have degree 0 for the filtration $F \cdot \mathcal{D}^{\wedge}$, so that we can define the filtration on each term in the standard way. Hence all horizontal maps of the diagram (9) respect the induced filtration on each term. Actually, we can show more: These sequences are even strict resolutions of $\left(\mathcal{G}^{\wedge}\right)^{r}$ resp. $\left(\Omega^{2} \otimes G(* D)\right)^{\wedge}$. Recall that a filtered complex $\left(\mathcal{K}^{\bullet}, F_{\bullet}, d\right)$ of coherent $\mathcal{D}$-modules is a strict resolution of a coherent $\mathcal{D}$-module $\mathcal{M}$ iff for any $k \in \mathbb{Z}$ the induced morphism $\left(F_{k} \mathcal{K} \bullet, d\right) \rightarrow F_{k} \mathcal{M}$ (the latter object seen as a complex concentrated in one degree) is a quasi-isomorphism. In order to check this property, it suffices to show that the induced morphism $\left(g r_{k}^{F} \mathcal{K}^{\bullet}, d\right) \rightarrow g r_{k}^{F} \mathcal{M}$ is a quasi-isomorphism provided that for any $i, F_{k} \mathcal{K}^{i}$ is a finite $F_{0} \mathcal{D}^{\wedge}$-module. This criterion for strictness follows from [Sch85, proposition 1.1.3 d)] (and goes back to [SKK73, proposition 3.2.7]), namely, the filtration $F_{\bullet} \mathcal{D}^{\wedge}$ is in fact Zariskian in the sense of [Sch85, definition 1.1.2 2)]. To show this, we remark that the ring $\mathcal{D}^{\wedge}$ can be identified with the ring of formal micro-differential operators on $S \times T^{*}$ (more precisely, consider the sheaf of formal micro-differential operators on $T^{*}\left(S \times T^{*}\right) \backslash T_{S \times T^{*}}^{*}\left(S \times T^{*}\right)$, and restrict it to the image of the section $d s: S \times T^{*} \rightarrow T^{*}\left(S \times T^{*}\right)$ so that it can be considered as a sheaf on $S \times T^{*}$ and take its global sections) via Fourier-Laplace transformation sending $\theta$ to $\partial_{s}^{-1}$ and $\partial_{\theta}$ to $-s$, and then the filtration $F_{\bullet}$ on $\mathcal{D}^{\wedge}$ is nothing but the filtration induced by the degree of (micro-)differential operators. Then the Zariskian property is shown in [Sch85, proposition 2.2.1]. Notice that the finiteness over $F_{0} \mathcal{D}^{\bullet}$ of each filtration step of any module in both of the horizontal exact sequences in diagram (9) obviously holds.

Let us first show that the graded object of the upper sequence in diagram (9) is acyclic. This graded complex is the Koszul complex of the symbols (with respect to $F_{\bullet} \mathcal{D}^{\wedge}$ ) of $P_{1}^{t}$ and $-P_{2}^{t}$ in $\operatorname{gr}_{\bullet} F^{(}\left(\mathcal{D}^{\wedge}\right)$, i.e., the Koszul complex of $(-\theta \cdot t \cdot v)^{n}-\frac{c}{n^{n}} t$ and $\theta^{2} \cdot u+n t \theta \cdot v$. The ideal generated by these two functions has codimension two, notice that

$$
\frac{\operatorname{gr}_{\bullet}^{F}\left(\mathcal{D}^{\wedge}\right)}{\left((-\theta \cdot t \cdot v)^{n}-\frac{c}{n^{n}} t, \theta^{2} \cdot u+n t \theta \cdot v\right)}=\frac{\operatorname{gr}_{\bullet}^{F}\left(\mathcal{D}^{\wedge}\right)}{\left((-\theta \cdot t \cdot v)^{n}-\frac{c}{n^{n}} t, u+\theta^{-1} \cdot n t \cdot v\right)} \cong \frac{\mathbb{C}[[\theta]]\left[\theta^{-1}, t, t^{-1}, v\right]}{\left((-\theta \cdot t \cdot v)^{n}-\frac{c}{n^{n}} t\right)}
$$

and the latter ring is obviously two-dimensional. Hence the symbols of $P_{1}^{t}$ and $-P_{2}^{t}$ define a complete intersection and thus form a regular sequence in $\operatorname{gr}_{\bullet}^{F}\left(\mathcal{D}^{\wedge}\right)$. It follows that the Koszul complex of these two functions is a resolution of the quotient ring. We conclude that the upper line of diagram (9) is a strict resolution of $\left(\mathcal{G}^{\wedge}\right)^{r}$.

A similar argument applies to the lower line of this diagram: The graded complex $\operatorname{gr}_{\bullet} F\left(\Omega^{\bullet}\left(\mathcal{D}^{\wedge}\right)\right)$ is a resolution of $\operatorname{gr}_{\bullet}^{F}\left(\left(\Omega^{2}\right)^{\wedge}\right)$ since it is simply the Koszul complex of the elements $u, v$ in the $\operatorname{ring} \operatorname{gr}_{\bullet}^{F}\left(\mathcal{D}^{\wedge}\right)=\mathbb{C}[[\theta]]\left[\theta^{-1}, t, t^{-1}, u, v\right]$. Hence the graded complex of $\left(\Omega^{\bullet}(\mathcal{D}) \otimes G(* D)\right)^{\wedge}$ is a resolution of $\operatorname{gr}_{\bullet}^{F}\left(\Omega^{2} \otimes G(* D)^{\wedge}\right)$ (since the differential on $\left(\Omega^{\bullet}(\mathcal{D}) \otimes\right.$ $G(* D))^{\wedge}$ is the identity on the second factor), so that $\left(\Omega^{\bullet}(\mathcal{D}) \otimes G(* D)\right)^{\wedge}$ is a strictly filtered resolution of $\left(\Omega^{2} \otimes G(* D)\right)^{\wedge}$. Moreover, the isomorphism $\left(\left(\Omega^{\bullet} \otimes \mathcal{D}\right) \otimes G(* D)\right)^{\wedge} \stackrel{\cong}{\rightrightarrows}\left(\left(\Omega^{\bullet} \otimes G(* D)\right) \otimes \mathcal{D}\right)^{\wedge}$ used in the proof of theorem 6 is strict because it is filtered and its inverse (induced by the isomorphism $(G(* D) \otimes \mathcal{D})^{\wedge} \cong\left(\mathcal{\cong} \otimes(G(* D))^{\wedge}\right)$ also respects the filtration. We conclude that the formal version of the complex (5), i.e., the lower line of diagram (9) is a strict resolution of $\left(\Omega^{2} \otimes G(* D)\right)^{\wedge}$.

We have now seen that both lines of this diagram are strict, and moreover that the rightmost isomorphism $\left(\varphi^{\wedge}\right)^{r}$ strictly shifts the filtration by -3 . Then the same holds for any of the vertical morphisms, and the induced isomorphism $\Psi^{\wedge}$ satisfies

$$
\Psi^{\wedge}:\left(F_{\bullet+3}^{\vee}\left(G(* D)^{\vee}\right)\right)^{\wedge} \stackrel{\cong}{\longrightarrow}\left(F_{\bullet}^{\mathbb{D}}(\mathbb{D} \mathcal{G})\right)^{\wedge} .
$$

where $F_{\bullet}^{\vee}\left(G(* D)^{\vee}\right):=\mathcal{H o m}_{\mathbb{C}\left[\theta, t, t^{-1}\right]}\left(F_{\bullet} G(* D), \mathbb{C}\left[\theta, t, t^{-1}\right]\right)$ and where $F_{\bullet} \mathbb{D}(\mathbb{D} \mathcal{G})$ is the filtration on $\mathbb{D} \mathcal{G}$ dual to $F_{\bullet} \mathcal{G}$ in the sense of [Sai88, 2.4]. Moreover, due to the strictness of the resolution of $\left(\mathcal{G}^{\wedge}\right)^{r}$, the (formal version of the) duality 
isomorphism from proposition 5 satisfies

$$
\Phi^{\wedge}:\left(\mathcal{G}^{\wedge}, F_{\bullet}^{\wedge}\right) \stackrel{\cong}{\longrightarrow} \iota^{*}\left((\mathbb{D} \mathcal{G})^{\wedge},\left(F_{\bullet-(n+2)}^{\mathbb{D}}\right)^{\wedge}\right),
$$

where again $F_{\bullet}^{\mathbb{D}}$ is the filtration dual to $F_{\bullet}$. Combining this with the isomorphism $\varphi^{\wedge}:\left(F_{0} \mathcal{G}\right)^{\wedge}=\mathcal{G}_{0}^{\wedge} \cong G_{0}(* D)^{\wedge}$, noticing that $F_{0}^{\vee}\left(G(* D)^{\vee}\right)=G_{0}(* D)^{\vee}$ and looking at the (formal version of the) diagram (7) we finally conclude that

$$
\Phi^{m e r, \wedge}=\left(\iota^{*} \Psi\right)^{\wedge} \circ \Phi^{\wedge} \circ\left(\varphi^{-1}\right)^{\wedge}: G_{0}(* D)^{\wedge} \stackrel{\cong}{\longrightarrow}\left(\iota^{*}\left(\theta^{n-1} \cdot G_{0}(* D)^{\vee}\right)\right)^{\wedge},
$$

as required.

\section{Limit and weak logarithmic Frobenius structures}

In this final section we indicate how the theorems 6 and 7 from the last section can be used to sharpen the results from [GMS09, section 4 and 5]. We start with a preliminary lemma. Consider the grading of $\mathbb{C}\left[\theta, \theta^{-1}, t, t^{-1}\right]$ for which $\operatorname{deg}(\theta)=1$ and $\operatorname{deg}(t)=n$. We obtain an induced grading on $\mathcal{D}$ with $\operatorname{deg}\left(\partial_{\theta}\right)=-1$ and $\operatorname{deg}\left(\partial_{t}\right)=-n$. Notice that this grading does neither induce the filtration $F_{\bullet}$ on $\mathcal{D}$ considered in the proof of theorem 7 nor the usual order filtration used in the proof of proposition 5. The module $G(* D)$ (as well as its various lattices) carries a compatible grading, we have $\operatorname{deg}\left(\omega_{i}\right)=i-1$ and the connection operator $\nabla$ on $G(* D)$ from proposition 3 as well as the isomorphism $\varphi$ from theorem 4 are homogenous of degree 0.

Lemma 8. (see also [GMS09, conjecture 5.5]) The pairing $S$ from theorem 6 is homogenous of degree 0 with respect to this natural grading of $(G(* D), \nabla)$, i.e., we have

$$
S\left(G(* D)_{k}, G(* D)_{l}\right) \subset \mathbb{C}\left[\theta, \theta^{-1}, t, t^{-1}\right]_{k+l} .
$$

Proof. The statement we need is equivalent to the fact that the isomorphism $\Phi^{\text {mer }}$ from the proof of theorem 6 is homogenous of degree 0 . In order to show this, we need again to consider the comparison isomorphism $\Psi$ : $(G(* D), \nabla)^{\vee} \rightarrow \mathbb{D}(G(* D))$ from the proof of theorem 6 . Recall that it was constructed from diagram (6), which we recall below. However, we will write it in such a way that all morphism are homogenous for the above grading. We have

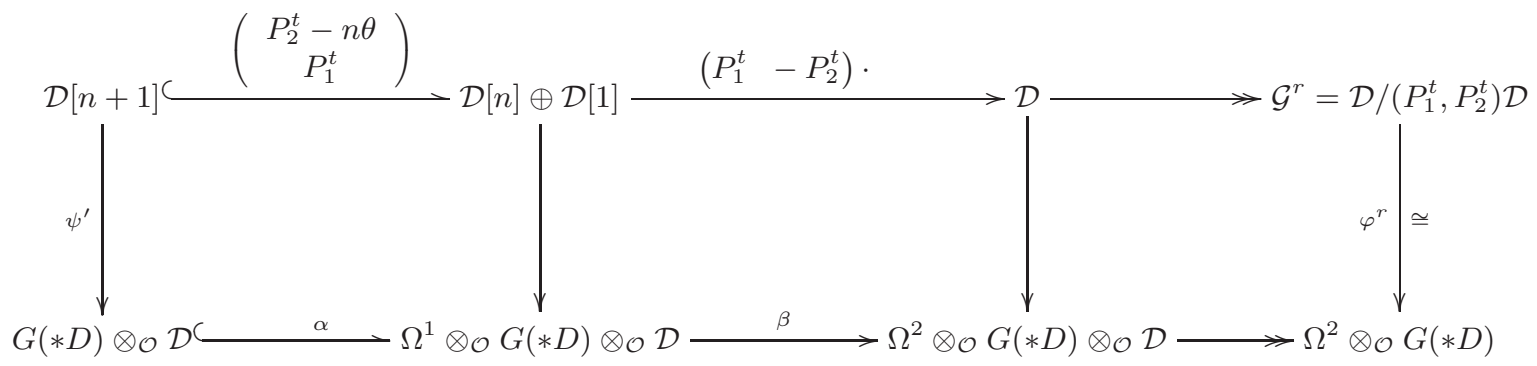

where $\mathcal{D}[k]$ is $\mathcal{D}$ with the shifted grading defined by $\mathcal{D}[k]_{l}=\mathcal{D}_{k-l}$. From the description of the morphisms $\alpha$ and $\beta$ given in the proof of theorem 6 we see that both are homogenous of degree 0 . Hence the above diagram is a morphism of 0 -graded complexes of right $\mathcal{D}$-modules (i.e., a morphism of complexes of graded right $\mathcal{D}$-modules with differentials of degree 0 ). Therefore the degrees of the vertical maps are all equal, and we only have to determine the degree of the rightmost morphism $\varphi^{r}$. Recall that it is defined by sending 1 to $(d \theta \times d t) \otimes(n$ vol $/ d h)$, hence, $\operatorname{deg}\left(\varphi^{r}\right)=n+1$. It follows that $\operatorname{deg}\left(\psi^{\prime}\right)=n+1$. The morphism $\psi: \mathcal{D} \rightarrow G(* D) \otimes_{\mathcal{O}} \mathcal{D}$ from diagram (6) is the same as $\psi^{\prime}$, but defined on $\mathcal{D}$ rather than on $\mathcal{D}[n+1]$, hence $\operatorname{deg}(\psi)=2 n+2$. It follows that we have $\operatorname{deg}(\Psi)=2 n+2$, where $\Psi: G(* D)^{\vee} \rightarrow \mathbb{D} \mathcal{G}$ is the isomorphism induced by the dual of $\psi$. Now consider again diagram $(7)$ from above. From $\operatorname{deg}(\varphi)=0$ and $\operatorname{deg}(\Phi)=\operatorname{deg}\left(\iota^{*} \Psi\right)=2 n+2$ we $\operatorname{deduce} \operatorname{deg}\left(\Phi^{\text {mer }}\right)=0$, as required.

As a consequence, we obtain a quite precise result on the behavior of the pairing $S$ with respect to the bases $\underline{\omega}$ and $\underline{\widetilde{\widetilde{\omega}}}$ from proposition 3 .

Theorem 9. Let $D \subset V$ be reductive and $f \in V^{\vee}$ generic. Then

1. The basis $\underline{\widetilde{\omega}}$ from proposition 3 yields $a\left(V^{+}, S\right)$-solution (in the sense of [DS03, appendix B.d]) to the Birkhoff problem in family of $\left(G_{0}(* D), \nabla\right)$ and more precisely, we have

$$
S\left(\widetilde{\omega}_{i}, \widetilde{\omega}_{j}\right) \in \mathbb{C} \cdot \theta^{n-1} \cdot t^{2 k} \cdot \delta_{i+j, n+1}
$$

for some $k \in \mathbb{N}$. 
2. The basis $\underline{\omega}$ from proposition 3 is a $\left(V^{+}, S\right)$-solution to the Birkhoff problem of $(G(\log D) / t \cdot G(\log D), \nabla)$ and more precisely, we have

$$
S\left(\omega_{i}, \omega_{j}\right) \in \mathbb{C} \cdot \theta^{n-1} \cdot \delta_{i+j, n+1}
$$

3. We have $S\left(G_{0}(\log D), G_{0}(\log D)\right) \subset \theta^{n-1} \mathbb{C}[\theta, t]$ (this is the second part of [GMS09, conjecture 5.5.]).

4. Put $\widehat{G}(* D):=\oplus_{i=1}^{n} \mathcal{O}_{\mathbb{P}^{1} \times T^{*}} \cdot \widetilde{\omega}_{i}$ and $\widehat{G}(\log D):=\oplus_{i=1}^{n} \mathcal{O}_{\mathbb{P}^{1} \times T} \cdot \omega_{i}$, then $S$ induces non-degenerate pairings

$$
S: \widehat{G}(* D) \otimes \iota^{*} \widehat{G}(* D) \rightarrow \mathcal{O}_{\mathbb{P}^{1} \times T^{*}}(-(n-1), n-1)
$$

and

$$
S:(\widehat{G}(\log D) / t \cdot \widehat{G}(\log D)) \otimes \iota^{*}(\widehat{G}(\log D) / t \cdot \widehat{G}(\log D)) \rightarrow \mathcal{O}_{\mathbb{P}^{1}}(-(n-1), n-1) .
$$

where $\mathcal{O}_{\mathbb{P}^{1} \times T^{*}}(a, b)$ (resp. $\left.\mathcal{O}_{\mathbb{P}^{1}}(a, b)\right)$ is the subsheaf of $\mathcal{O}_{\mathbb{P}^{1} \times T^{*}}\left(*\{0, \infty\} \times T^{*}\right)$ (resp. $\mathcal{O}_{\mathbb{P}^{1}}(*\{0, \infty\})$ of meromorphic functions with a pole of order a along $\{0\} \times T^{*}$ (resp. at 0 ) and a pole of order $b$ along $\{\infty\} \times T^{*}$ (resp. at $\infty)$.

Proof. It was shown in [GMS09, corollary 4.12] that $\underline{\widetilde{\omega}}$ (resp. $\underline{\omega}$ ) is a $V^{+}$-solution for $\left(G_{0}(* D), \nabla\right)$ (resp. for $\left.\left(G_{0}(\log D) / t \cdot G_{0}(\log D), \nabla\right)\right)$. Recall from [DS03, appendix B.d] (see also [GMS09, lemma-definition 4.6]) that $\underline{\widetilde{\omega}}$ (resp. $\underline{\omega})$ is compatible with $S$ (i.e., a $S$-solution) iff $S\left(\widetilde{\omega}_{i}, \widetilde{\omega}_{j}\right) \in \theta^{n-1} \cdot \mathbb{C}\left[t, t^{-1}\right]$ for all $i \in\{1, \ldots, n\}\left(\right.$ resp. $S\left(\omega_{i}, \omega_{j}\right) \in \theta^{n-1} \cdot \mathbb{C}$ for all $i \in\{1, \ldots, n\})$.

In order to show the statements 1. and 2., we use the proof of [GMS09, theorem 4.13], where an additional hypothesis on the multiplicity of the smallest spectral number $\nu_{\sigma(1)}$ was made. However, as $\operatorname{deg}\left(\omega_{i}\right)=i-1(\operatorname{resp} \cdot \operatorname{deg}(\widetilde{\omega})=i-1+k \cdot n$ for some $k \in \mathbb{N}$ ) we deduce from the theorems 6 and 7 as well as from lemma 8 that whenever we take $i \in\{1, \ldots, n\}$ such that $\nu_{i}=\nu_{\sigma(1)}$, then $S\left(\omega_{i}, \omega_{k}\right) \in \mathbb{C} \cdot \delta_{i+j, n+1} \cdot \theta^{n-1}$ and $S\left(\widetilde{\omega}_{i}, \widetilde{\omega}_{k}\right) \in \mathbb{C} \cdot \theta^{n-1} \cdot t^{2 k} \cdot \delta_{i+j, n+1}$. Then the proof of theorem 4.13 in loc.cit. shows that this is true for any $i \in\{1, \ldots, n\}$, that is, we obtain the formulas (11) and (12), but also the statements 3 . and 4 from above.

We can now give the promised application of the above results. It consist of a sharpening of theorems 5.1, 5.7 and 5.9 from [GMS09]. We we do not need anymore to make an assumption on the multiplicity of the spectral number at infinity of $f_{\mid h^{-1}(t)}$ and we also know that [GMS09, conjecture 5.5.] holds. Using this, the proofs of the theorems below are the same as in loc.cit. and are therefore omitted. We always suppose that $D$ is reductive and $f \in V^{\vee}$ generic.

The first result is the construction of a Frobenius manifold structure on a miniversal deformation space of the restriction $f_{\mid h^{-1}(t)}$.

Theorem 10. [GMS09, theorem 5.1] Consider a semi-universal unfolding $F: B_{t} \times M_{t} \rightarrow D_{\delta}$ as in loc.cit. theorem 5.1 (where $D_{\delta} \subset \mathbb{C}$ is a small disc and $B_{t}=h^{-1}(t) \cap B_{\epsilon}$, with $M_{t}$ and $B_{\epsilon}$ being balls in $\mathbb{C}^{n}$ ). Then (any non-zero multiple of) any of the section $\omega_{i}$ from proposition 3 is primitive and homogenous and induces a Frobenius structure on $M_{t}$.

The next result gives the construction of a Frobenius structure at $t=0$, that one may see as associated to the restriction of $f$ to $D$.

Theorem 11. (see also [GMS09, theorem 5.7]) The germ at the origin of the $\mathcal{R}_{h}$-miniversal deformation space of $f$ (called $M_{0}$ ) carries a constant Frobenius structure (i.e., such that the structure constant of the multiplication are constant in flat coordinates).

Finally, we also obtain the structure of a "weak logarithmic Frobenius manifold" associated to the couple $(f, h)$ (see [Rei09] for the definition of a logarithmic Frobenius manifold and [GMS09, definition 5.8] for a variant called "weak logarithmic").

Theorem 12. (see also [GMS09, theorem 5.9]) The module $G^{\prime}$ from loc.cit., theorem 5.9 underly a weak log $\Sigma$ trTLEP-structure and the form $t^{-k} \widetilde{\omega}_{i}=t^{-k} \omega_{i}^{(3)}$ (where $i$ is the index from loc.cit., lemma 5.3) is primitive and homogenous. It yields a weak logarithmic Frobenius manifold associated to $(f, h)$.

\section{References}

[BM06] Ragnar-Olaf Buchweitz and David Mond, Linear free divisors and quiver representations, Singularities and computer algebra (Cambridge) (Christoph Lossen and Gerhard Pfister, eds.), London Math. Soc. Lecture Note Ser., vol. 324, Cambridge Univ. Press, 2006, Papers from the conference held at the University of Kaiserslautern, Kaiserslautern, October 18-20, 2004, pp. 41-77. 
[Bri09] Tom Bridgeland, Spaces of stability conditions, Algebraic geometry-Seattle 2005. Part 1 (D. Abramovich, A. Bertram, L. Katzarkov, R. Pandharipande, and M. Thaddeus, eds.), Proc. Sympos. Pure Math., vol. 80, Amer. Math. Soc., Providence, RI, 2009, Papers from the AMS Summer Research Institute held at the University of Washington, Seattle, WA, July 25-August 12, 2005, pp. 1-21.

[DM09] Antoine Douai and Etienne Mann, The small quantum cohomology of a weighted projective space, a mirror $\mathcal{D}$-module and their classical limits, Preprint math.AG/0909.4063, to appear in Geometrica Dedicata (doi: 10.1007/s10711-012-9768-3), 2009.

[Dou09] Antoine Douai, A canonical Frobenius structure, Math. Z. 261 (2009), no. 3, 625-648.

[DS03] Antoine Douai and Claude Sabbah, Gauss-Manin systems, Brieskorn lattices and Frobenius structures. I, Ann. Inst. Fourier (Grenoble) 53 (2003), no. 4, 1055-1116.

[GMNS09] Michel Granger, David Mond, Alicia Nieto, and Mathias Schulze, Linear free divisors and the global logarithmic comparison theorem., Ann. Inst. Fourier (Grenoble) 59 (2009), no. 1, 811-850.

[GMS09] Ignacio de Gregorio, David Mond, and Christian Sevenheck, Linear free divisors and Frobenius manifolds, Compositio Mathematica 145 (2009), no. 5, 1305-1350.

[GS10] Michel Granger and Mathias Schulze, On the symmetry of b-functions of linear free divisors, Publ. Res. Inst. Math. Sci. 46 (2010), no. 3, 479-506.

[Rei09] Thomas Reichelt, A construction of Frobenius manifolds with logarithmic poles and applications, Comm. Math. Phys. 287 (2009), no. 3, 1145-1187.

[Sai88] Morihiko Saito, Modules de Hodge polarisables, Publ. Res. Inst. Math. Sci. 24 (1988), no. 6, 849-995 (1989).

[Sai89] _ _ On the structure of Brieskorn lattice, Ann. Inst. Fourier (Grenoble) 39 (1989), no. 1, $27-72$.

[Sch85] Pierre Schapira, Microdifferential systems in the complex domain, Grundlehren der Mathematischen Wissenschaften [Fundamental Principles of Mathematical Sciences], vol. 269, Springer-Verlag, Berlin, 1985.

[Sev11] Christian Sevenheck, Bernstein polynomials and spectral numbers for linear free divisors, Ann. Inst. Fourier (Grenoble) 61 (2011), no. 1, 379-400.

[SKK73] Mikio Sato, Takahiro Kawai, and Masaki Kashiwara, Microfunctions and pseudo-differential equations, Hyperfunctions and pseudo-differential equations (Proc. Conf., Katata, 1971; dedicated to the memory of André Martineau), Lecture Notes in Mathematics, Vol. 287, Springer, Berlin, 1973, Dedicated to the memory of André Martineau, Edited by Hikasaburo Komatsu, pp. 265-529. Lecture Notes in Math., Vol. 287.

[SST00] Mutsumi Saito, Bernd Sturmfels, and Nobuki Takayama, Gröbner deformations of hypergeometric differential equations, Algorithms and Computation in Mathematics, vol. 6, Springer-Verlag, Berlin, 2000.

[Tak05] Atsushi Takahashi, Matrix factorizations and representations of quivers I, Preprint math.AG/0506347, 2005 .

Lehrstuhl für Mathematik VI

Institut für Mathematik

Universität Mannheim, A 5, 6

68131 Mannheim

Germany

Christian.Sevenheck@math.uni-mannheim.de 